\title{
LA MUSICALITÉ EST-ELLE UN HÉRITAGE DE NOTRE HISTOIRE BIOLOGIQUE ?
}

Une approche comparative et multidisciplinaire

Carla Aimé, Mathilde Le Covec, Dalila Bovet et Rana Esseily

Presses Universitaires de France | «nfance »

2020/1 N 1 | pages 41 à 66

ISSN 0013-7545

ISBN 9782130823155

Article disponible en ligne à l'adresse :

https://www.cairn.info/revue-enfance-2020-1-page-41.htm

Distribution électronique Cairn.info pour Presses Universitaires de France.

(C) Presses Universitaires de France. Tous droits réservés pour tous pays.

La reproduction ou représentation de cet article, notamment par photocopie, n'est autorisée que dans les limites des conditions générales d'utilisation du site ou, le cas échéant, des conditions générales de la licence souscrite par votre établissement. Toute autre reproduction ou représentation, en tout ou partie, sous quelque forme et de quelque manière que ce soit, est interdite sauf accord préalable et écrit de l'éditeur, en dehors des cas prévus par la législation en vigueur en France. Il est précisé que son stockage dans une base de données est également interdit. 


\title{
La musicalité est-elle un héritage de notre histoire biologique ? Une approche comparative et multidisciplinaire
}

\author{
Carla Aimé, Mathilde Le Covec, Dalila Bovet, Rana Esseily ${ }^{a}$
}

\begin{abstract}
RÉSUMÉ
La musicalité (i.e. l'ensemble des capacités associées à la perception, à l'appréciation et la production musicale) est largement répandue chez notre espèce. Selon certains chercheurs, elle aurait évolué car elle favoriserait la survie et/ou la reproduction. Ainsi, les origines biologiques de la musicalité ont été étudiées dans la littérature, chez l'espèce humaine mais aussi chez d'autres espèces. Les études suggèrent des fonctions adaptatives, dont l'attractivité en tant que partenaire de reproduction, la communication mère-bébé, ou encore la cohésion des groupes sociaux. Dans cette revue comparative, nous présenterons les données existantes sur la musicalité chez les espèces humaine et non-humaines pour chacune des fonctions évoquées ci-dessus. Nous discuterons ensuite la convergence évolutive de la musicalité chez certaines espèces ainsi que son origine biologique.

MOTS-CLÉS : MUSICALITÉ, ÉVOLUTION, ADAPTATION, INTERACTIONS SOCIALES, REVUE COMPARATIVE.
\end{abstract}

\section{ABSTRACT}

\section{Is musicality a heritage of our evolutionary history?}

A comparative and pluridisciplinary approach

Musicality (i.e. the set of cognitive abilities associated with music perception, appreciation and production) is widely shared among humans. Some researchers have suggested that it may have evolved because of some implications on survival and/or reproduction. Thus, the biological roots of musicality in human and non-human species have been studied in the literature, suggesting potential adaptive functions such as attractiveness as a partner for reproduction, mother-infant communication and social cohesion. In this comparative review, we present studies on musicality both in human and in non-human species in each of the aforemen-

a Laboratoire Éthologie Cognition Développement. Université Paris Nanterre. 200 Avenue de la République, 92000 Nanterre.E-mails : mme.carla.aime@gmail.com; dalila.bovet@parisnanterre.fr; mathilde.le_covec@hotmail.com; rana.esseily@parisnanterre.fr. 
tioned adaptive functions. We then discuss convergent evolution in some species and address the question of innateness of musical abilities.

KEYWORDS: MUSIC, EVOLUTION, ADAPTATION, SOCIAL INTERACTIONS, COMPARATIVE REVIEW.

\section{INTRODUCTION}

Chez notre espèce, Homo sapiens, nous savons que la musique existe depuis au minimum 35,000 ans avant notre ère. En effet, cela correspond à la datation du plus ancien instrument connu : la flûte de Hohle Fels (Conard et al., 2009). Cependant, on peut imaginer que les véritables origines de la musique soient bien plus anciennes car d'autres formes ne nécessitant pas d'instruments, et donc laissant beaucoup moins de traces archéologiques (chant, tambourinage en rythme sur des troncs ou avec des cailloux, etc.), pourraient avoir préexisté. Dans cette revue, nous nous intéresserons aux origines de la musicalité, définie comme l'ensemble des capacités cognitives associées à la perception, à l'appréciation et/ou à la production musicale (Hoeschele et al., 2015).

Plusieurs hypothèses ont été suggérées dans la littérature concernant les origines des capacités musicales humaines. Notamment, plusieurs auteurs (Darwin, 1871 ; Roederer, 1984 ; Dissanayake, 2000 ; Miller, 2000 ; Dunbar, 2017) ont suggéré que la musicalité pourrait avoir une origine biologique et évolutive : elle pourrait avoir été favorisée au cours de l'évolution car elle apporterait un avantage adaptatif aux individus, c'est-à-dire un avantage en termes de survie et/ou de succès reproducteur (Darwin, 1871). Cependant, les positions de ces auteurs divergent concernant la nature de l'avantage adaptatif qui pourrait être associé aux capacités musicales. Nous passerons ici en revue les différentes hypothèses proposées, puis nous aborderons les indices expérimentaux en faveur d'une origine évolutive de la musique. Notamment, nous nous intéresserons d'abord à la question de l'innéité des capacités musicales, puis à celle de la relation entre musicalité et survie et/ou succès reproducteur. Nous aborderons chacune de ces questions non seulement chez l'humain, mais aussi chez d'autres espèces animales. En effet, démontrer l'existence chez d'autres espèces de capacités qualifiées chez l'humain de «musicales » serait un indice supplémentaire en faveur de leur origine biologique et évolutive, plutôt que simplement culturelle. De plus, s'intéresser à l'évolution de la musicalité chez d'autres groupes du vivant pourrait nous aider à mieux comprendre les fonctions adaptatives de la musique chez l'humain.

C'est dans ce souci comparatif que nous nous concentrerons sur la notion de musicalité plutôt que sur celle de musique. En effet, bien que certains auteurs n'hésitent pas à faire un parallèle entre la musique humaine et les chants produits par les oiseaux (évoquant des similarités structurelles et des influences comparables sur les émotions des congénères, Rothenberg et al., 2014), l'utilisa- 
tion de ce terme pour décrire la perception et/ou la production de sons par des animaux non humains ne fait cependant pas consensus. Le terme «musique» renvoie notamment à une connotation culturelle : la musique est souvent considérée comme un construit social et culturel basé sur la musicalité (Honing et al., 2015 ; Trehub et al., 2015). A l'inverse, comme mentionné précédemment, la musicalité est définie comme l'ensemble des capacités cognitives associées à la perception, à l'appréciation et/ou à la production musicale (Hoeschele et al., 2015). Or, certaines capacités correspondant à cette définition chez l'humain semblent également présentes chez d'autres espèces, comme nous le détaillerons dans les prochains paragraphes.

Nous nous intéresserons donc ici à la musicalité : est-elle innée ? Quelles sont ses origines et pourquoi la retrouve-t-on dans toutes les populations humaines? Est-elle un héritage de notre évolution et, si oui, en quoi pourraitelle être adaptative ? A-t-elle évolué chez plusieurs groupes au cours de l'histoire du vivant? Avec le même avantage adaptatif ? Telles sont les questions auxquelles nous tenterons de répondre en croisant les regards de différentes disciplines : la biologie évolutive, la psychologie du développement et l'éthologie. Cette approche multidimensionnelle permettra d'apporter un éclairage approfondi à ces questions, en considérant à la fois les mécanismes, les fonctions et le développement de la musicalité chez les humains et les autres animaux.

\section{HYPOTHÈSES SUR LES ORIGINES ÉVOLUTIVES DE LA MUSICALITÉ}

Comme mentionné précédemment, la musique est un art ancestral chez l'humain. De plus, elle existe dans toutes les cultures (Brown et Jordania, 2013) et les capacités qui y sont associées semblent en partie innées. Des études génétiques ont en effet identifié dans l'ADN humain plusieurs variantes associés aux capacités musicales (Ukkola et al., 2013). De plus, comme nous le verrons par la suite, de nombreuses études montrent que certaines capacités de perception, d'appréciation voire de production musicale existent dès le plus jeune âge et sont partagées avec d'autres espèces animales. Des prédispositions biologiques à la perception et à l'appréciation, voire à la production musicale pourraient donc exister, menant à s'interroger sur une potentielle origine évolutive de la musicalité.

Plusieurs auteurs ont évoqué cette hypothèse, le premier étant Darwin en 1871. Il suggérait que nos capacités musicales pourraient jouer un rôle dans l'attractivité ressentie vis à vis d'un partenaire de reproduction, et ainsi avoir évolué par sélection sexuelle. En d'autres termes, parce qu'elles favoriseraient l'accès aux partenaires sexuels, les capacités musicales seraient corrélées avec le succès reproducteur, et donc adaptatives (Darwin, 1871 ; Miller, 2000). De son côté, Dissanayake (2000) suggère que la musicalité aurait plutôt évolué dans le 
cadre de la communication mère-bébé, car elle favoriserait la communication des émotions et ainsi le lien maternel et les chances de survie du bébé. En effet, la musicalité pourrait être un véritable moyen de communication des émotions, via la voix mais aussi la synchronisation des mouvements (voir aussi Malloch, 1999 ; Malloch et Trevarthen, 2009). D'autres auteurs ont émis l'idée que la musique favoriserait plus largement les liens sociaux et la cohésion du groupe, et ainsi la survie des individus qui le composent (Roederer, 1984; Freeman, 1998 ; Dunbar, 2017). Par exemple, selon Freeman (1998), l'écoute de musique pourrait placer les individus dans de meilleures dispositions pour coopérer avec autrui, en modifiant leur état émotionnel par des mécanismes neuro-hormonaux. Par ailleurs, en tant qu'activité traditionnellement collective nécessitant des interactions fortes entre les individus, la production musicale pourrait participer à créer et entretenir les liens sociaux spécifiquement au sein du groupe dont les membres partagent cette expérience (Roederer, 1984). Enfin, Dunbar (2017) va plus loin en suggérant que la musique puis le langage pourraient avoir remplacé le toilettage mutuel comme "ciment social», à une période de notre histoire où celui-ci aurait pris trop de temps pour assurer la cohésion de groupes sociaux devenant de plus en plus larges. Le langage et la musique semblent d'ailleurs partager certaines caractéristiques : ils sont aussi efficaces l'un que l'autre pour communiquer des émotions, et les signaux accoustiques associés à chaque émotion y sont similaires (Scherer, 1991; Juslin et Laukka, 2003). Néanmoins, il est important de signaler que l'idée d'une origine adaptative de la musicalité n'a pas toujours fait l'unanimité dans la littérature scientifique. Notamment, Pinker (1997) défend l'idée d'une origine exclusivement culturelle. Pour lui, les capacités associées à la perception et à la production de la musique auraient évolué car elles sont toutes impliquées dans d'autres fonctions. La musique serait ensuite apparue comme une simple activité récréative, sans lien avec la survie ou la reproduction, qui pourrait disparaître sans aucun effet sur l'évolution humaine.

\section{LA MUSICALITÉ EST-ELLE INNÉE?}

La perception de la musique est basée sur un set de mécanismes cognitifs et perceptifs qui interagissent. Notamment, deux traits essentiels à la perception musicale sont la capacité à percevoir les notes (perception tonale), ainsi que celle de percevoir la temporalité d'une séquence sonore (groupement, rythme et mesure). Nous aborderons dans les paragraphes suivants les études relatives à ces deux traits chez le bébé humain ainsi que chez des espèces non humaines. En effet, trouver des capacités de perception musicale présentes dès le plus jeune âge, et partagées entre différentes espèces, suggérerait une origine de la musicalité au moins en partie biologique, plutôt que seulement culturelle. 


\section{La perception tonale}

L'innéité de cette capacité est supportée par de nombreuses études portant sur la musicalité chez les bébés humains, ainsi que sur la perception de la musique par d'autres espèces. Elles montrent notamment que la perception des notes chez les bébés est très similaire à celle des adultes (Trehub, 2003). Par exemple, les bébés comme les adultes reconnaissent une mélodie même lorsque la tonalité est déplacée vers le haut ou vers le bas (Trehub, 2003). Par ailleurs, la perception des notes pourrait avoir une très longue histoire évolutive puisque certains mécanismes impliqués pourraient être partagés avec, au moins, la majorité des vertébrés (Ravignani et al., 2014 ; Fitch, 2006). En effet, la perception d'une mélodie serait sous-tendue par plusieurs stratégies perceptives (notamment l'oreille relative et l'oreille absolue) chez l'être humain comme chez d'autres espèces (Hulse et al., 1992).

\section{Capacité de discrimination tonale}

Les différences tonales entre deux mélodies peuvent concerner la structure générale de ces mélodies (le contour), ou les liens entre les différentes notes (les intervalles). Chez l'humain, le contour est un indicateur puissant puisque les bébés peuvent détecter un changement de contour même lorsque les deux stimuli leurs sont présentés à 15 secondes d'intervalle (Trehub, 2001). Le contour mélodique est également l'attribut le plus saillant dans le langage adressé au bébé (Fernald et Mazzie, 1991). Les bébés reconnaissent aussi une mélodie quand le contour mélodique ne change pas mais qu'il y a une transposition globale d'une tonalité au-dessus ou en dessous de la mélodie originale (Chang et Trehub, 1977). De plus, lorsqu'on maintient constants le contour mélodique, les bébés sont capables de percevoir un changement d'un demi-ton dans une triade de notes ascendantes (Trehub et al., 1986). Enfin, dans certains cas, les capacités des bébés à détecter de tels changements dépassent même celles des adultes (notamment dans les mélodies non diatoniques, où les bébés occidentaux sont capables de percevoir les changements de tonalité même lorsque ceux-ci modifient le contour mélodique, Trehub, 2003).

Quant aux autres espèces animales, la discrimination d'accords, de mélodies et de morceaux a été montrée chez plusieurs d'entre elles. Par exemple, les pigeons (Columba livia) peuvent différencier un morceau de Bach d'un morceau d'Hindermith et d'un autre de Stravinsky (Porter et Neuringer, 1984), et les poissons rouges (Carassius auratus) distinguent deux morceaux de musique ('’un de Bach et l'autre de Stravinski ; Shinozuka et al., 2013). Certains animaux non humains sont même capables de généraliser ces différences à deux compositeurs ou deux styles de musique. En effet, les rats font la distinction entre un morceau de Bach et un autre de Stravinski grâce à un conditionnement opérant, mais peuvent aussi généraliser cette discrimination entre les deux compositeurs à d'autres de leurs morceaux (Otsuka et al., 2009). Quant aux pigeons, ils généralisent la discrimination entre Bach et Stravinski à d'autres compositeurs de 
musique baroque versus moderne (Porter et Neuringer, 1984). Enfin, les carpes (Cyprinus carpio), après avoir appris à discriminer la musique d'un compositeur de blues (John Lee Hooker) de celle d'un compositeur de musique classique (Bach), généralisent cette distinction entre les styles à d'autres artistes (Chase, 2001). Chez les oiseaux chanteurs et les perroquets, plusieurs espèces montrent même des préférences musicales individuelles. C'est notamment le cas des paddas de Java (Lonchura oryzivora) qui se posent sur un perchoir diffusant un compositeur plutôt qu'un autre (Bach versus Schoenberg; Watanabe et Nemoto, 1998), ou encore des perruches calopsittes (Nymphicus Hollandicus) qui, face à un écran tactile leur laissant le choix entre déclencher différents morceaux de musique, révèlent également des préférences individuelles (Le Covec et al., soumis à Animal Cognition). Cependant, le rôle du contour mélodique dans la discrimination entre plusieurs morceaux chez les espèces non humaines n'est pas toujours clair : la plupart du temps, il n'a pas été spécifiquement étudié, et lorsque c'est le cas, les résultats diffèrent selon les espèces. Par exemple, une éléphante Elephas maximus s'est montrée capable de distinguer deux mélodies, voire deux morceaux de musique, en se basant sur plusieurs caractéristiques dont la tonalité (Reinert, 1957). Au contraire, bien que les rats soient effectivement capables de distinguer plusieurs morceaux de musique, ils se basent pour cela sur le timbre mais pas sur la mélodie (Rattus norvegicus, Poli et Previde, 1991). Comme nous allons le voir par la suite, nous savons par contre que plusieurs espèces distinguent les mélodies sur la base des intervalles entre les notes.

\section{Capacité à discriminer la consonance de la dissonance}

Les intervalles représentent la distance entre deux notes successives. Souvent, les bébés confondent des mélodies qui partagent le même contour mais avec des intervalles différents (Trehub et al., 1986, 1990) sauf si ces intervalles répondent à certaines règles. Par exemple, ils peuvent détecter un changement d'un demi-ton si le ratio entre les notes est petit (Cohen et al., 1987; Trainor et Trehub, 1993). Par ailleurs, certains intervalles de notes sont généralement perçus par les humains comme plus agréables et sont qualifiés de consonants, par opposition aux intervalles dissonants, généralement considérés comme désagréables (Trainor et Heinmiller, 1998). Cette distinction est démontrée chez les bébés de 2 , 4 et 6 mois, qui préfèrent écouter des mélodies consonantes comparées aux mélodies dissonantes et présentent des affects négatifs en présence de stimuli dissonants (Trainor et al; 2002 ; Trainor et Heinmiller, 1998 ; Zentner et Kagan, 1998). Plantinga et Trehub (2014) suggèrent que ce résultat pourrait être simplement dû à une exposition plus fréquente des bébés à la musique consonante. En effet, ils montrent que les bébés de 6 mois écoutent significativement plus une musique qui leur est familière, peu importe qu'elle soit consonante ou dissonante. Cependant, Masataka (2006) montre une préférence pour la musique consonante chez des bébés de deux jours seulement y compris s'ils sont nés de parents sourds, ce qui suggère néanmoins une préfé- 
rence innée pour les intervalles consonants. De plus, des études montrent que les intervalles consonants ou les séquences de notes séparées par de petits ratios sont plus facilement encodés par les bébés (comme par les enfants et les adultes) que les intervalles dissonants ou les séquences de notes séparées par de larges ratios (Schellenberg et Trehub, 1994, 1996b; Trainor, 1997). Les bébés de 9 mois peuvent également percevoir des changements dans les intervalles si ceux-ci sont consonants mais pas s'ils sont dissonants (Schellenberg et Trehub, 1996a). Il faut noter cependant une part de variation culturelle concernant l'appréciation de la consonance et de la dissonance chez l'humain, puisque McDermott et al. (2016) montrent une indifférence à la dissonance chez des indiens d'Amazonie.

Enfin, concernant les espèces non humaines, plusieurs d'entre elles peuvent distinguer des accords consonants ou dissonants, comme les macaques japonais (Macaca fuscata; Izumi, 2000) et les étourneaux (Sturnus vulgaris; Hulse et al., 1995). Ces derniers peuvent en plus transférer cette distinction à d'autres accords dont les fréquences de base sont différentes.

\section{La perception de la temporalité dans la musique}

La perception temporelle implique que les individus doivent pouvoir percevoir les unités et les organiser entre elles. Selon Trehub et Hannon (2006), trois aspects temporels sont fondamentaux en musique : le groupement, le rythme (organisation structurelle du temps), et la mesure (régularité périodique). Les capacités de perception temporelle des bébés sont globalement similaires à celles de l'adulte, avec cependant quelques particularités.

\section{Groupement et organisation rythmique}

Les bébés et les enfants groupent des séquences de notes, comme les adultes, sur la base de leur similarité en termes de tonalité, d'intensité et de timbre (Thorpe et Trehub, 1989; Thorpe et al., 1988). Un autre critère de groupement saillant est le ton descendant à la fin d'une phrase musicale (Jusczyk et Krumhansl, 1993). En ce qui concerne le rythme, les bébés comme les adultes utilisent la taille et l'ordre des intervalles temporels pour discriminer différents patterns. De manière analogue à la transposition tonale, les bébés reconnaissent une séquence de notes lorsque le rythme est altéré à condition que les durées relatives restent les mêmes (Trehub et Thorpe, 1989). À l'âge de 2 mois, les bébés qui ont été habitués à un rythme montrent un intérêt pour un ordre différent des intervalles temporels (Demany et al., 1977). Puis, entre 4 et 10 mois, les bébés montrent une réaction à la nouveauté en cas de réarrangement des durées (Chang et Trehub, 1977 ; Lewkowicz, 2003).

D'autres espèces que la nôtre semblent également sensibles au rythme. C’est le cas des perroquets gris du Gabon (Psittacus erithacus) qui face à un écran tactile leur laissant le choix entre déclencher un morceau de musique rythmé ou un morceau de musique calme, ont montré des préférences stables mais 
différentes selon les individus (Péron et al., 2012). Deux choucas ont également appris à discriminer deux signaux rythmiques différents (Corvus monedula ; Reinert, 1965).

\section{Perception de la mesure et capacité de synchronisation}

La mesure permet aux individus de se synchroniser avec une musique (danser, marcher, taper ou chanter en rythme). Elle permet aussi d'anticiper les évènements futurs (Jones et Boltz, 1989). A 10 mois, les bébés perçoivent des changements de durée et d'intensité dans les mesures imposées par un musicien (Palmer et al., 2001). À 7 mois, les bébés familiarisés avec trois groupes de rythmes identiques induisant une même mesure écoutent plus longtemps un nouveau rythme induisant une nouvelle mesure qu'un nouveau rythme induisant la même mesure (Hannon et Johnson, 2005). De plus, les bébés utilisent des indices de mouvement pour inférer les mesures. Par exemple, des bébés âgés de 7 mois bercés sur un rythme ambigu montrent une préférence pour le rythme induisant la mesure du bercement (Pillips-Silver et Trainor, 2005). Dans certains cas, les bébés dépassent même les capacités des adultes à détecter des changements de mesure, notamment pour les musiques non-isochrones (i.e. dont la pulsation n'est pas régulière). Ces capacités disparaissent vers l'âge de 12 mois, probablement du fait de l'exposition à des mesures plus standards dans la musique occidentale (Hannon et Trehub, 2005).

Chez l'animal non humain, la perception de la mesure n'est pas toujours simple à évaluer et a très peu été étudiée. Cependant quelques rares études s'y sont penchées et ont montré des éléments suggérant que certaines espèces y seraient sensibles. Notamment, les étourneaux (Hulse et al., 1985), les diamants mandarins (Taeniopygia guttata) et les perruches ondulées (Melopsittacus undulatus, Hoeschele et Bowling, 2016) distinguent des pulsations régulières de pulsations irrégulières. De plus, plusieurs espèces d'oiseaux montrent des comportements de parade supposant une certaine capacité à produire une pulsation régulière (voir par exemple Ota et al. 2015 ; Soma et Mori, 2015). Plus largement, plusieurs études ont montré des comportements partageant cette régularité temporelle : un mâle chimpanzé captif par exemple, Barney, a spontanément produit une pulsation bimanuelle (Dufour et al., 2015), un éléphant d'Asie a frappé un tempo régulier sur un instrument de musique (Patel et Iversen, 2006), les cacatoès noirs créent des outils avec lesquels ils tapent en rythme sur des troncs suivant des styles individuels (Heinsohn et al., 2017), et les calopsittes et les corbeaux à gros bec (Corvus macrorynchos) tambourinent des séquences avec leur bec (Le Covec et al., soumis à Animal Cognition). La capacité de synchronisation à une pulsation a également été montrée chez une otarie de Californie (Zalophus californianus, Cook et al., 2013), chez des perruches ondulées (Melopsittacus undulatus, Hasegawa et al., 2011), ainsi que chez des macaques (Macaca mulatta) et un chimpanzé (Zarco et al., 2009). Cependant macaques et chimpanzés nécessitent un très long apprentissage (jusqu'à un an) avant d'y parvenir et ils le font avec moins de précision et plus de variabilité temporelle que les humains (Zarco et 
al., 2009). Par contre, au moins six espèces d'oiseaux psittaciformes (perroquets et perruches) sont spontanément capables de danser en rythme (Fitch, 2009 ; Schachner et al., 2009) suivant un large panel de tempos (Patel et al., 2009).

En résumé, il existe de nombreuses similarités entre les espèces non humaines, les bébés humains et les humains adultes concernant la perception des traits musicaux. Ces similarités suggèrent une origine biologique de la musicalité et une prédisposition innée à percevoir la musique. Notamment, il semble peu probable que les capacités perceptives musicales des bébés proviennent de l'exposition pré et postnatale à l'environnement acoustique, pour plusieurs raisons. D’abord, comme nous l'avons mentionné, les bébés dépassent les adultes sur certaines compétences et ce n'est que vers l'âge de 5-7 ans que les enfants deviennent sensibles aux spécificités culturelles de leur musique. Ensuite, les bébés nés de parents sourds-muets, exposés à la langue des signes, présentent les mêmes similarités perceptives que les bébés nés de parents entendants (Masataka, 2006). Cependant, dans une revue de la littérature sur la perception de la musique chez les bébés, les adultes et les espèces non humaines, Trehub et Hannon (2006) suggèrent que les capacités perceptives des bébés seraient le produit de mécanismes généraux de traitement de signaux acoustiques qui ne sont pas propres à l'humain ni spécifiques à la musique. Par exemple, les notes descendantes et l'allongement de la durée jouent un rôle important dans la segmentation des mots et sont particulièrement exagérés dans le langage destiné aux bébés (Fernald, 1991). La perception rythmique du langage serait également un critère de segmentation de la parole (Ramus et al., 1999) et est prise en compte lors de discrimination de différentes langues (Nazzi et al., 1998). Les auteurs argumentent que ceci ne s'oppose pas à une base biologique de la perception musicale, puisque ce n'est pas la perception des traits musicaux en soi qui donnerait à la musique un ancrage biologique, mais les conséquences sociales de l'utilisation de la musique dans les rituels, la communication et le soin qui font d'elle une partie essentielle de la condition humaine et de sa survie. Il reste donc à identifier les avantages adaptatifs qui pourraient être associés à la musicalité. Les prochains paragraphes seront consacrés aux différentes théories à ce sujet et aux indices expérimentaux les corroborant ou non.

\section{MUSICALITÉ ET SÉLECTION SEXUELLE}

Comme mentionné précédemment, Darwin fut le premier à suggérer, dans The descent of man (1871), que la musicalité humaine pourrait avoir une origine adaptative. Il défendait la thèse selon laquelle ces capacités auraient évolué par sélection sexuelle. Dans la théorie de l'évolution (Darwin, 1859), la sélection sexuelle est présentée comme le deuxième moteur de l'adaptation des espèces, au même titre que la sélection naturelle. Si cette dernière fait référence à la sélection des traits associés à une meilleure survie ou une meilleure fertilité, la 
sélection sexuelle concerne de son côté les traits favorisant l'accès aux partenaires pour la reproduction. Ainsi, si de meilleures capacités musicales étaient associées à une plus grande attractivité pour les partenaires potentiels, elles seraient corrélées à un meilleur succès reproducteur et pourraient donc être adaptatives. On pourrait notamment imaginer que de bonnes capacités musicales indiquent de bonnes capacités perceptives (audition, discrimination des sons) mais aussi motrices (coordination), créatives, d'apprentissage et de mémoire, et enfin sociales (car la musique est traditionnellement une activité de groupe). Ainsi, une plus grande attractivité des individus musiciens aurait d'abord été sélectionnée car les choisir comme partenaire offrirait l'assurance de transmettre de «bons gènes » à la descendance. La musicalité elle-même aurait ainsi pu augmenter à son tour en fréquence dans les populations par sélection sexuelle. Cette hypothèse a également été reprise par Miller (2000).

\section{Performances vocales et rythmiques chez les oiseaux}

Les liens entre musicalité et attractivité sexuelle sont surtout bien connus dans le domaine de l'éthologie, en particulier chez les spécialistes du chant des oiseaux. Traditionnellement, le champ lexical de la musique est librement appliqué à certaines de leur vocalisations, qualifiées de « chants » par les scientifiques comme par les musicologues. Il a été d'ailleurs été démontré que beaucoup d'oiseaux chantent selon des règles précises, similaires à celles qui gouvernent la musique humaine et amènent les humains à ressentir des émotions lors de l'écoute (Rothenberg et al., 2014). Notamment, tout comme les humains, les oiseaux tendent à réduire la monotonie de leurs chants. Il semble également que les humains sous-estiment souvent les aptitudes perceptives des oiseaux, puisque ceux-ci sont capables de percevoir plus de détails que nous sur le plan auditif. Leur résolution temporelle des sons complexes est, par exemple, plus précise que la nôtre (Dooling et al., 2002). Or chez les oiseaux, les chants mais aussi les rythmes et les enchaînements de mouvements associés sont généralement liés à des comportements de séduction et apparaissent majoritairement chez les mâles pendant la saison des parades (voir par exemple Searcy et Andersson, 1986). Certains oiseaux montrent par exemple, lorsqu'ils font la cour, des comportements qualifiés de danses ou de chorégraphies, suivant des séquences complexes et spécifiques. C'est le cas du mâle ménure superbe (Menura novaehollandiae; Dalziell et al., 2013), des manakins à col d'or (Manacus vitellinus; Coccon et al., 2012), ou encore des cordons bleus cyanocéphales (Uraeginthus cyanocephalus; Ota et al., 2015). D'autres espèces produisent des chants suivant une syntaxe précise, tels que les mâles paddas de Java (Lonchura oryzivora), qui coordonnent leurs vocalisations avec des claquements de leur bec (Soma et Mori, 2015). Enfin, certaines productions rythmiques paraissent également intervenir dans un cadre de parade : les mâles cacatoès noirs fabriquent des baguettes de bois avec lesquels ils produisent des pulsations régulières dont les femelles semblent être l'auditoire (Heinsohn et al., 2017), et les mâles calopsittes élégantes tambourinent avec leur bec selon des tempos 
réguliers (Le Covec et al., soumis à Animal Cognition). Ce comportement est majoritairement observé en période de reproduction (Le Covec et al., 2019). Hormis une étude chez l'étourneau sansonnet (Sturnus vulgaris) montrant une utilisation du chant comme marqueur d'appartenance à un groupe (Hausberger et al., 2008), c'est donc principalement dans le cadre théorique de la sélection sexuelle que les fonctions adaptatives du chant et de la musicalité chez les oiseaux ont jusqu'à présent été étudiées. La complexité du chant, la possession d'un répertoire varié et la qualité des productions rythmiques associées renseigneraient les partenaires ainsi que les compétiteurs potentiels sur la qualité individuelle de l'émetteur (Gil et Gahr, 2002). Par exemple, les mâles produisant des chants complexes auraient un succès reproducteur plus élevé, car ils seraient à la fois plus attractifs pour les femelles et plus intimidants pour les rivaux (Andersson, 1994). La complexité du chant, la perception de ses caractéristiques précises et la préférence des femelles pour les chants complexes seraient donc des traits favorisés par l'évolution darwinienne.

\section{Musicalité et sélection sexuelle chez l'être humain}

La sélection sexuelle semble donc bien avoir joué un rôle essentiel dans l'évolution de la musicalité chez les oiseaux. Mais en est-il de même concernant l'évolution de la musicalité chez l'humain? Les résultats expérimentaux sont beaucoup moins édifiants chez l'espèce humaine même si on peut imaginer que les rituels dans les tribus ont pu être l'occasion pour évaluer les performances musicales des partenaires sociaux. Cependant, il n'existe pas à notre connaissance de preuves expérimentales d'une corrélation entre capacités musicales et attractivité chez l'humain, malgré quelques tentatives infructueuses de la mettre en évidence (Mosing et al., 2014, 2015). L'hypothèse de l'évolution de la musicalité par sélection sexuelle chez l'humain manque donc, du moins pour l'instant, de preuves expérimentales convaincantes (Fitch, 2005). Par ailleurs, il faut noter que même chez les oiseaux, signaler sa qualité aux partenaires et compétiteurs potentiels pourrait ne pas suffire à expliquer l'émergence du chant et de la musicalité. En effet, Odom et al. (2014) ont montré que, malgré le fait que ce soit généralement elles qui choisissent leur partenaire de reproduction, le chant chez les femelles oiseaux chanteurs n'est pas aussi rare que ce que l'on croyait auparavant (celui-ci est présent chez $71 \%$ des 1141 espèces considérées dans leur méta-analyse). Cette étude affirme également, sur la base d'analyses phylogénétiques, que mâles et femelles chantaient chez l'ancêtre commun des oscines (oiseaux chanteurs) modernes. Bien qu'elle ne remette pas en question l'idée d'une influence de la sélection sexuelle sur la complexité et les caractéristiques précises du chant chez les oiseaux, elle encourage donc à s'interroger sur d'autres mécanismes potentiels impliqués dans l'émergence du chant et de la musicalité. 


\section{MuSICALITÉ ET COMMUNICATION MÈRE-ENFANT}

D'après Dissanayake (2000), la musicalité pourrait avoir évolué dans le cadre de la relation mère-bébé, car elle favoriserait la communication des émotions et ainsi le lien maternel. Il existe une seule étude à notre connaissance concernant les liens entre musicalité et vocalisations maternelles chez des espèces non humaines. Celle-ci montre que chez les Saïmiris, les mères utilisent certains types de cris exclusivement avec leur petit pouvant être associés au langage adressé au bébé (Biben et al., 1988). Concernant l'humain, la littérature en psychologie du développement nous fournit de nombreux indices en faveur de cette hypothèse. Notamment, des études mentionnées ci-après soutiennent l'idée que la musicalité jouerait un rôle essentiel dans la communication des émotions entre une mère et son bébé.

Premièrement, bien que la parole ne soit pas de la musique en soi, on peut noter que le langage adressé aux bébés (LAB) présente certaines caractéristiques l'en rapprochant telles que la répétition, la rythmicité, l'élongation des voyelles et des contours mélodiques (Fernald, 1992 ; Papousek, 1992). En particulier, il semblerait que la répétition soit importante : lors de l'analyse du langage adressé au bébé, Bergeson et Trehub (2002) montrent que chaque mère a une signature vocale unique et que la plupart des mères conservent une stabilité de la mélodie avec les mêmes intervalles quand elles répètent leur discours. Par ailleurs, il existe une corrélation positive entre l'engagement émotionnel et le taux de répétition de la mélodie et de la phrase. La répétition se trouve également dans les mélodies populaires et dans les poèmes, signe d'expressivité émotionnelle. Deuxièmement, au-delà du langage, les mères interagissent souvent avec leur bébé en chantant (Trehub et al., 1997), et ceci est vrai dans toutes les cultures (Trehub et Trainor, 1998). Les chants adressés aux bébés ont des caractéristiques communes aux quatre coins du monde, si bien qu'ils sont aisément reconnaissables par un auditeur ne comprenant pas la langue utilisée : ils sont répétitifs, et ont une rythmicité et un contour mélodique très simples (Trehub et al., 1993 ; Unyk et al., 1992). Ces chants sont aussi caractérisés par leur haute expressivité émotionnelle. Les mères adoptent un ton plus élevé et un tempo plus lent lorsque leur chant est adressé à leur bébé (Trainor, 1996 ; Trehub et al., 1993,1997). En général, le ton élevé est associé à de la joie (Scherer, 1986; Murray et Arnott, 1993) et le tempo lent à de l'affection et de la tendresse (Fonagy et Magdics, 1963; Davitz, 1964). Comme pour le $\mathrm{LAB}$, le chant adressé au bébé est caractérisé par un petit répertoire de chansons répétées sans changement de style, de tempo ou de ton sauf quand le bébé est fatigué (Trehub et al., 1997). Cette stabilité permettrait de consolider la mémoire et de favoriser une humeur constante (Trehub et Nakata, 2001). Ainsi, l'effet positif du chant maternel sur le bébé (réduction de pleurs, apaisement, endormissement) augmenterait les chances que les mères chantent de nouveau pour le bébé (Trehub et al., 2015). Dès la naissance (Masataka, 1999) et à 6 mois (Trainor, 1996) les bébés prêtent plus attention au chant adressé 
au bébé qu'au chant non adressé au bébé, indiquant probablement une préférence pour des stimuli émotionnels. Enfin, Nakata et Trehub (2004) ont montré à des bébés de 6 mois des vidéos de leur mère soit qui chantait soit qui parlait. Les bébés ont regardé plus longtemps quand leur mère chantait et ont significativement réduit leur activité corporelle (mouvement). Cette différence n'est pas observée lorsque les bébés étaient exposés à un chant ou une parole adressée par une mère étrangère. Il existe aussi des preuves que le chant améliore les paramètres physiologiques des bébés prématurés (Saliba et al., 2018).

La musicalité semble donc être un moyen efficace de communication des émotions et d'influence sur le comportement, notamment utilisé par les mères s'adressant à leur bébé (les pères et d'autres membres de la famille chantent moins souvent que les mères, Trehub et al., 1997). Cette idée est également défendue par Malloch (1999), ou encore Malloch et Trevarthen (2009), qui parlent de «musicalité communicative ». Cela pourrait avoir constitué un avantage adaptatif favorisant l'émergence et l'évolution de la musicalité, comme suggéré par Dissanayake (2000). Par ailleurs, parallèlement ou dans un deuxième temps, une influence plus large (non limitée aux interactions mèrebébé) de la musique sur les comportements sociaux aurait également pu avoir joué un rôle primordial dans la perpétuité de la musique, comme l'ont suggéré plusieurs auteurs. Cette hypothèse fera l'objet de la partie suivante.

\section{MUSICALITÉ ET COMPORTEMENTS PROSOCIAUX}

Au-delà de la relation mère-enfant et de la sélection sexuelle, comme mentionné en introduction, certains auteurs ont suggéré que la musicalité favoriserait plus largement les interactions sociales, et ainsi la cohésion des groupes sociaux (Freeman, 1998 ; Roederer, 1984 ; Dunbar, 2017). En effet, la musique en groupe est à la base de nombreuses activités sociales dans les populations traditionnelles (rituels, cérémonies, etc.), et plusieurs études montrent notamment que la synchronisation rythmique favoriserait le lien social (Tarr et al., 2014, Cirelli et al., 2014).

Chez l'humain, c'est encore du côté de la psychologie du développement que l'on retrouve les premiers indices expérimentaux appuyant l'hypothèse d'une relation entre musique et comportements sociaux. Notamment, Kirschner et Tomasello (2010) ont montré que faire un jeu impliquant la production conjointe de musique, plutôt qu'un jeu non musical, favorise la coopération et l'entraide chez des dyades d'enfants de quatre ans. De plus, d'après Rabinowitch et al. (2013), une pratique musicale régulière favoriserait aussi l'empathie chez les 8-11 ans. D'après Good et Russo (2016), participer régulièrement à une activité de chant choral favoriserait davantage la coopération (mesurée par une version adaptée du dilemme du prisonnier) chez les enfants d'école élémentaire que participer à une activité d'arts plastiques en groupe ou à des jeux de compétition. Chez l’adulte, quelques études expérimentales 
montrent aussi un effet positif de l'écoute de musique sur la coopération (Kniffin et al., 2017), l'altruisme (Fukui et Toyoshima, 2014), et l'entraide (Fried et Berkowitz 1979 ; North et al., 2004). Il existe aussi une étude inter-culturelle révélant que, dans au moins quatre cultures différentes (au Kenya, aux Philippines, en Nouvelle-Zélande et en Allemagne), écouter de la musique au sein de la famille ou d'un groupe d'amis contribue à la cohésion sociale du groupe partageant cette expérience (Boer et Abubakar, 2014). Enfin, plusieurs études montrent un effet positif de la synchronisation rythmique (avec ou sans musique) sur la prosocialité (Tarr et al., 2014, Cirelli et al., 2014, 2016, 2017). Nous reviendrons plus loin sur la question de la synchronisation et des autres mécanismes potentiellement impliqués dans la relation entre musique et comportements sociaux.

Chez les animaux non humains, l'influence de la musique sur les comportements interactifs a peu été étudiée. On peut tout de même noter que chez de nombreuses espèces d'oiseaux, les duos vocaux peuvent renforcer les liens au sein du couple (Sonnenschein et Reyer, 1983 ; Hall, 2009). C'est par exemple le cas chez des perroquets tels que les amazones à nuque d'or (Dahlin et Whright, 2012). Chez certains singes monogames, comme les gibbons, on retrouve aussi des "chants » en duo permettant de favoriser les liens au sein du couple ou de la famille (Geissmann et Orgeldinger, 2000). Par ailleurs, sur un plan neurologique, chez la souris, l'exposition à la musique module l'activité de l'hippocampe (Angelucci et al. 2007), une structure cérébrale qui joue un rôle majeur dans le traitement des émotions, y compris les émotions sociales (Immordino-Yang et Singh 2013). Enfin, il a été montré que les chimpanzés de laboratoire avaient plus de comportements affiliatifs à l'écoute de musique instrumentale et moins de comportements agonistiques à l'écoute de musique vocale (Videan et al. 2007). Quant aux gorilles (Gorilla gorilla gorilla) en zoo, ils agressent moins leurs congénères quand ils écoutent de la musique classique (Wells et al. 2006).

\section{CONCLUSION ET PERSPECTIVES : QUID DES MÉCANISMES IMPLIQUÉS DANS LA RELATION ENTRE MUSIQUE ET COMPORTEMENTS SOCIAUX?}

Les études mentionnées nous révèlent donc que plusieurs des capacités qualifiées chez l'humain de "musicales " (notamment la perception tonale, la perception de la temporalité dans la musique ou encore la capacité à se synchroniser avec une pulsation) peuvent s'observer chez d'autres espèces que la nôtre. De plus, chez l'humain, la musicalité est présente dès le plus jeune âge et semble donc au moins en partie innée. Enfin, concernant les avantages adaptatifs qui pourraient expliquer l'émergence et l'évolution de la musicalité, l'hypothèse de la sélection sexuelle manque de preuves concrètes. Par contre, la musique semble être un moyen efficace de communiquer des émotions et 
d'influencer les comportements, à la fois dans le cadre de la relation mère-bébé (Dissanayake, 2000) et dans les relations sociales de manière plus générale. Comme suggéré par Freeman (1998), Roederer (1984), Merker (2000) ou encore Dunbar (2017), la musicalité pourrait avoir évolué car la musique favoriserait la cohésion des groupes sociaux. Cette hypothèse est corroborée par la littérature à la fois en éthologie et en psychologie du développement, comme détaillé précédemment. Elle l'est également par nos précédents travaux, qui montrent un effet positif de l'écoute de musique sur les interactions sociales à la fois chez les enfants d'âge préscolaire et chez une espèce d'oiseau, les perruches callopsittes. En effet, en 2017, nous avions testé 144 enfants dans deux écoles maternelles et montré qu'il y avait davantage d'interactions physiques et verbales lors d'une tâche de coopération (i.e. tirer sur des ficelles de manière synchronisée pour actionner un tiroir et accéder à une récompense) entre les enfants ayant écouté au préalable sept minutes de musique consonante par rapport à ceux ayant écouté un bruit contrôle. Concernant les perruches callopsittes, nous avions également montré qu'il y avait davantage de proximité spatiale chez un groupe de douze individus lors de l'écoute de musique (consonante ou dissonante), et moins de comportements agressifs lors de l'écoute de musique consonante par rapport à de la musique dissonante. Mettre ainsi en évidence un même avantage associé à la musicalité à la fois chez les humains et chez les oiseaux renforce l'idée que la musicalité pourrait avoir une origine évolutive. La musicalité pourrait en effet avoir évolué chez les deux groupes avec la même fonction adaptative : favoriser les interactions sociales positives et la cohésion des groupes sociaux. En effet, il faut rappeler ici que les capacités musicales semblent plus limitées chez les primates non humains (McDermott et Hauser, 2006 ; Zarco et al., 2009 ; Honing et al., 2012), pourtant phylogénétiquement plus proches de nous que les oiseaux. La musicalité ne semble donc pas être un trait ancestral, qui serait partagé par les humains et les oiseaux car hérité d'un ancêtre commun. C'est pourquoi nous suggérons plutôt que la musicalité pourrait avoir évolué séparément chez les humains et chez les oiseaux, à des moments différents de leur histoire évolutive. En biologie évolutive, on parle alors d'évolution convergente.

Cependant, de nombreuses questions restent ouvertes, notamment en ce qui concerne les mécanismes impliqués dans cette relation entre musique et comportements sociaux chez les humains et chez les oiseaux. Quel sont les mécanismes qui sous-tendent la musique et qui peuvent expliquer son influence sur les comportements pro-sociaux? Ces mécanismes sont-ils universels? Sont-ils spécifiques à la musique ou s'étendent-ils à d'autres domaines ? Ce sont autant de sujets qui restent à explorer. De nombreux mécanismes pourraient-être impliqués, mais nous ne détaillerons ici que trois d'entre eux : la répétition, la synchronisation et les émotions. Dans les paragraphes suivants, nous aborderons ces mécanismes, leur universalité et leur spécificité. 


\section{La répétition}

La répétition est un trait musical universel (Trehub, 2003) qui apporterait un cadre stable à toute interaction sociale. Elle permet d'anticiper les évènements et ainsi de pouvoir y participer (Trehub et Hannon, 2006). La répétition favorise également la mémorisation et la reconnaissance des signaux musicaux d'une part et de celui ou celle qui les produit d'autre part (Trehub et Nakata, 2001). Par exemple, les mères chantent à leur bébé avec une signature vocale spécifique et de manière très répétitive, ce qui est probablement très utile pour la reconnaissance vocale et ainsi la survie de l'enfant. Dans toutes les cultures, les unités ou les phrases musicales ne dépassent pas sept notes par octave et sont identifiées grâce à leur répétition et leur redondance, avec ou sans variations. D'ailleurs, c'est l'un des traits les plus saillants lorsque la mère s'adresse à son petit en chantant et, de manière intéressante, il existe une corrélation positive entre le taux d'engagement émotionnel de la mère et le taux de répétition (Trehub, 2003). De même, dans le chant des oiseaux, des syllabes ou des motifs plus complexes sont fréquemment répétés (Baptista et Keister, 2005), et cette répétition joue un rôle dans l'attractivité du chant : par exemple, les canaris produisent une phrase dite sexy, car particulièrement appréciée des femelles ; cette phrase est composée de syllabes répétées de façon très rapide (Leboucher et al., 2012). La répétition pourrait donc conférer à la musique une valeur adaptative importante.

\section{La synchronisation}

La synchronisation est un deuxième mécanisme sous-jacent à la musique qui pourrait favoriser les interactions sociales. Elle est possible grâce aux régularités périodiques appelées mesures et perçues comme des temps forts ou faibles d'un évènement musical (Trehub et Hannon, 2006). La mesure permet aux individus d'un groupe de synchroniser leurs mouvements (en dansant, marchant, tapant, etc.) à travers toutes les cultures. Elle est saillante chez les bébés dès l'âge de 7 mois (Hannon et Johnson, 2005), et permet de guider l'attention de manière dynamique et d'anticiper de futurs évènements (Jones et Boltz, 1989). Chez les oiseaux, le cacatoès Snowball synchronise ses mouvements avec la musique qu'il entend selon un répertoire comprenant 14 mouvements, dont deux composés (Keehn et al., 2019). Il a été suggéré que ce comportement était social, car il permettait à l'oiseau d'interagir avec les membres de son groupe (en l'occurrence, les humains qui s'occupaient de lui). L'action synchronisée est donc considérée comme un bon candidat pour expliquer l'effet de la musique sur la cohésion du groupe et les interactions sociales. Benzon (2005) suggère que la musique activerait un réseau neuronal et cordonnerait le flux temporel de l'activité neuronale à la fois chez les individus et entre les individus, participant à produire ou à écouter de la musique. En effet, les adultes ayant bougé ensemble en synchronie s'apprécient plus, coopèrent et s'aident plus que ceux qui bougent ensemble mais de manière asynchrone. Les études chez les 
enfants montrent que des enfants âgés de 4 ans ayant bougé en synchronie avec un adulte sont plus aidants que des enfants ayant bougé de manière asynchrone (Tunçgenç et Cohen, 2016; Krischner et Tomasello, 2010). Des enfants âgés de 8 ans qui participent à une activité de chant choral (sous-tendant donc une synchronie) sont plus coopératifs que ceux qui participent à une activité d'art plastique (Good et Russo, 2016), et ceux qui tapent un rythme en synchronie avec un pair le jugent plus proche d'eux et plus similaire qu'un autre pair avec qui ils ont tapé de manière asynchrone (Rabonowitch et Knafo-Noam, 2015). Chez les bébés, il existe de plus en plus d'études sur la synchronie et son effet sur les comportements prosociaux (voir Cirelli, 2018 pour une revue de la littérature). Ces études montrent qu'à partir de 12 mois, les bébés montrent une préférence pour un partenaire avec qui ils ont bougé de manière synchrone versus asynchrone (Tunçgenç et al., 2015) et qu'à 14 mois, les bébés aident plus l'expérimentateur qui a préalablement bougé avec eux en synchronie qu'un expérimentateur qui a bougé de manière asynchrone (en présence ou en absence de musique, Cirelli et al., 2014, 2016, 2017). A 16 mois, les bébés imitent d'avantage un adulte qui a été synchrone avec eux dans une première phase de jeu par rapport à un adulte qui a simplement joué avec eux sans synchroniser leurs actions (Somogyi et Esseily, 2013).

\section{L'émotion}

Un troisième mécanisme sous-jacent à la musique est l'émotion. Chez l'animal non-humain, l'influence de la musique sur les émotions a été étudiée à plusieurs reprises, que ce soit par l'intermédiaire de marqueurs physiologiques ou via les comportements exprimés par l'individu. Par exemple, l'écoute de musique déclenche une augmentation de la synthèse de calcium et de dopamine chez les rats, suggérant que cela pourrait diminuer les symptômes des maladies impliquant des dysfonctionnements dopaminergiques (Sutoo et Akiyama, 2004). Chez les chiens (Canus lupus familiaris), la musique diminue les aboiements, augmente le temps passé à se reposer (Wells et al., 2002) et engendre plusieurs changements physiologiques bénéfiques impliquant une diminution du niveau de stress (Bowman et al., 2017) : c'est spécialement le cas pour la musique classique (Kogan et al., 2012). Parmi les animaux captifs, les éléphants d'Asie montrent également une diminution des comportements stéréotypés à l'écoute de musique (Wells et Irwin, 2008) et les chimpanzés davantage de comportements sociaux tels que le toilettage, le jeu et l'exploration (Howell et al., 2003). Il est cependant intéressant de noter que tous les types de musique n'induisent pas forcément les mêmes effets en fonction des individus et des espèces concernés : il a par exemple été montré que la musique classique augmentait les comportements de peur chez les poules (Campo et al., 2005) et que les gorilles montraient plus de stéréotypies à la diffusion de rock et de musique classique (Robbins et Margulis, 2014).

Chez les humains adultes, plusieurs études ont montré que différents types de musique activent différentes aires cérébrales impliquées dans le traitement 
de la valence émotionnelle (Koelsch et al., 2006 ; Schmidt et Trainor, 2001). Une seule étude expérimentale a exploré l'émotion musicale chez les bébés et a montré que comme les adultes, les bébés étaient capables d'associer des extraits musicaux avec des photos représentant des expressions émotionnelles (Nawrot, 2003). L'émotion musicale a également été étudiée chez les bébés à travers le chant dirigé vers le bébé dans les interactions mère-bébé. Ces études montrent que le chant maternel est hautement émotionnel (Shenfield et al., 2003 ; Trehub, 2019) et affecte les émotions et l'attention des bébés (Trehub et Nakata, 2001). Ainsi, la musique étant toujours ancrée dans un contexte social émotionnel, il est tout à fait raisonnable de penser qu'elle influence les choix et les comportements des individus dans des contextes d'interactions sociales.

\section{Mécanismes généraux ou mécanismes spécifiques ?}

L'existence de similarités dans le traitement de la musique au niveau tonal et temporal entre les espèces humaines et non humaines suggère que les capacités de musicalité seraient le produit de mécanismes généraux de traitement des signaux qui ne sont spécifiques ni à la musique ni à l'humain. En effet, certains de ces mécanismes sont largement partagés avec d'autres capacités telles que le langage (Trehub et Hannon, 2006). Ceci n'est pas opposé à l'idée d'une base biologique de la musique. L'universalité de la musique dans les rituels et le soin met en évidence l'importance de la musique comme une partie essentielle de la condition humaine plutôt qu'un produit dérivé ; des mécanismes généraux peuvent sous-tendre la perception de la musique mais des mécanismes motivationnels spécifiques sont nécessaires pour expliquer comment la musique a été perpétuée dans toutes les cultures humaines. Trehub et Hannon (2006) suggèrent que la musique peut utiliser des systèmes déjà existants afin de servir des fonctions sociales importantes et est, de ce point de vue, adaptative.

\section{RÉFÉRENCES}

Andersson, M.B. (1994). Sexual selection. Princeton University Press.

Angelucci, F., Ricci, E., Padua, L., Sabino, A., Tonali, P.A. (2007). Music exposure differentially alters the levels of brain-derived neurotrophic factor and nerve growth factor in the mouse hypothalamus. Neuroscience letters, 429(2-3), 152-155.

Baptista L.F., R.A. Keister. (2005). Why birdsong is sometimes like music. Perspectives in Biology and Medicine, 48(3), 426-443.

Benzon, W.L. (2005). Synch, Song, and Society. Human Nature Review, 5, 66-86.

Bergeson, T.R., Trehub, S.E. (2002). Absolute pitch and tempo in mothers' songs to infants. Psychological Science, 13(1), 72-75.

Boer, D., Abubakar, A. (2014). Music listening in families and peer groups: benefits for young people's social cohesion and emotional well-being across four cultures. Frontiers in Psychology, 5, 392. 
Bowman, A., Scottish, S.P.C.A., Dowell, F.J., Evans, N.P. (2017). The effect of different genres of music on the stress levels of kennelled dogs. Physiology \& behavior, 171, 207-215.

Brown, S., Jordania, J. (2013). Universals in the world's musics. Psychology of Music, 41(2), 229-248.

Campo, J.L., Gil, M.G., Davila, S.G. (2005). Effects of specific noise and music stimuli on stress and fear levels of laying hens of several breeds. Applied Animal Behaviour Science, 91(1-2), 75-84.

Chang, H.W., Trehub, S.E. (1977). Infants' perception of temporal grouping in auditory patterns. Child development, 1666-1670.

Chase, A.R. (2001). Music discriminations by carp (Cyprinus carpio). Animal Learning \& Behavior, 29(4), 336-353.

Cirelli, L.K. (2018). How interpersonal synchrony facilitates early prosocial behavior. Current opinion in psychology, 20, 35-39.

Cirelli, L.K., Einarson, K.M., Trainor, L.J. (2014). Interpersonal synchrony increases prosocial behavior in infants. Developmental science, 17(6), 1003-1011.

Cirelli, L.K., Wan, S.J., Spinelli, C., Trainor, L.J. (2017). Effects of Interpersonal Movement Synchrony on Infant Helping Behaviors: Is Music Necessary?. Music Perception: An Interdisciplinary Journal, 34(3), 319-326.

Cirelli, L.K., Wan, S.J., Trainor, L.J. (2016). Social effects of movement synchrony: increased infant helpfulness only transfers to affiliates of synchronously moving partners. Infancy, 21(6), 807-821.

Coccon, F., Schlinger, B.A., Fusani, L. (2012). Male Golden-collared Manakins Manacus vitellinus do not adapt their courtship display to spatial alteration of their court. Ibis, 154(1), 173-176.

Cohen, A.J., Thorpe, L.A., Trehub, S.E. (1987). Infants' perception of musical relations in short transposed tone sequences. Canadian Journal of Psychology/Revue canadienne de psychologie, 41(1), 33.

Conard, N.J., Malina, M., Münzel, S.C. (2009). New flutes document the earliest musical tradition in southwestern Germany. Nature, 460(7256), 737.

Cook, P., Rouse, A., Wilson, M., Reichmuth, C. (2013). A California sea lion (Zalophus californianus) can keep the beat: motor entrainment to rhythmic auditory stimuli in a non-vocal mimic. Journal of Comparative Psychology, 127(4), 412.

Dahlin, C.R., Wright, T.F. (2012). Duet function in the yellow-naped amazon, Amazona auropalliata: evidence from playbacks of duets and solos. Etho$\log y, 118(1), 95-105$.

Dalziell, A.H., Peters, R.A., Cockburn, A., Dorland, A.D., Maisey, A.C., Magrath, R.D. (2013). Dance choreography is coordinated with song repertoire in a complex avian display. Current Biology, 23(12), 1132-1135.

Darwin, C. (1859). On the origins of species by means of natural selection. London: Murray.

Darwin, C. (1871). The descent of man and selection in relation to sex. London: Murray.

Davitz, J.R. (1964). The communication of emotional meaning. New York: McGraw-Hill.

Demany, L., McKenzie, B., Vurpillot, E. (1977). Rhythm perception in early infancy. Nature, 266(5604), 718-719.

Dissanayake, E. (2000). Antecedents of the temporal arts in early mother-infant interaction. The origins of music (pp. 389-410). Cambridge, MA: MIT Press. 
Dooling, R.J., Leek, M.R., Gleich, O., Dent, M.L. (2002). Auditory temporal resolution in birds: discrimination of harmonic complexes. The Journal of the Acoustical Society of America, 112(2), 748-759.

Dufour, V., Poulin, N., Curé, C., Sterck, E.H. (2015). Chimpanzee drumming: a spontaneous performance with characteristics of human musical drumming. Scientific reports, 5, 11320.

Dunbar, R.I.M. (2017). Group size, vocal grooming and the origins of language. Psychonomic bulletin \& review, 24(1), 209-212.

Fernald, A. (1991). Prosody in speech to children: Prelinguistic and linguistic functions. Annals of Child Development, 8, 43-80.

Fernald, A., Mazzie, C. (1991). Prosody and focus in speech to infants and adults. Developmental psychology, 27(2), 209.

Fitch, W.T. (2005). The evolution of music in comparative perspective. Ann NY Acad Sci, 1060, 29-49.

Fitch, W.T. (2006). The biology and evolution of music: A comparative perspective. Cognition, 100(1), 173-215.

Fitch, W.T. (2009). Biology of music: another one bites the dust. Current Biology, 19(10), R403-R404.

Fonagy, I., Magdics, K. (1963). Emotional patterns in intonation and music. STUFLanguage Typology and Universals, 16(1-4), 293-326.

Freeman, W.J. (2000). A Neurobiological Role of Music in Social Bonding. In N.L. Wallin, B. Merker, S. Brown (eds.), The Origins of Music (pp. 411-424). Cambridge, MA: MIT Press.

Fried, R., Berkowitz, L. (1979). Music Hath Charms... And Can Influence Helpfulness. Journal of Applied Social Psychology, 9(3), 199-208.

Fukui, H., Toyoshima, K. (2014). Chill-inducing music enhances altruism in humans. Frontiers in Psychology, 5, 1215.

Geissmann, T., Orgeldinger, M. (2000). The relationship between duet songs and pair bonds in siamangs, Hylobates syndactylus. Animal Behaviour, 60(6), 805-809.

Gil, D., Gahr, M. (2002). The honesty of bird song: multiple constraints for multiple traits. Trends in Ecology \& Evolution, 17(3), 133-141.

Good, A., Russo, F.A. (2016). Singing promotes cooperation in a diverse group of children. Social Psychology.

Hall, M.L. (2009). A review of vocal duetting in birds. Advances in the Study of Behavior, 40, 67-121.

Hannon, E.E., Johnson, S.P. (2005). Infants use meter to categorize rhythms and melodies: Implications for musical structure learning. Cognitive psychology, 50(4), 354-377.

Hannon, E.E., Trehub, S.E. (2005). Tuning in to musical rhythms: Infants learn more readily than adults. Proceedings of the National Academy of Sciences, 102(35), 12639-12643.

Hasegawa, A., Okanoya, K., Hasegawa, T., Seki, Y. (2011). Rhythmic synchronization tapping to an audio-visual metronome in budgerigars. Scientific reports, 1, 120.

Hausberger, M., Bigot, E., Clergeau, P. (2008). Dialect use in large assemblies: a study in European starling Sturnus vulgaris roosts. Journal of avian biology, 39(6), 672-682. 
Heinsohn, R., Zdenek, C.N., Cunningham, R.B., Endler, J.A., Langmore, N.E. (2017). Tool-assisted rhythmic drumming in palm cockatoos shares key elements of human instrumental music. Science advances, 3(6), e1602399.

Hoeschele, M., Bowling, D.L. (2016). Sex differences in rhythmic preferences in the budgerigar (Melopsittacus undulatus): a comparative study with humans. Frontiers in Psychology, 7, 1543.

Hoeschele, M., Merchant, H., Kikuchi, Y., Hattori, Y., Ten Cate, C. (2015). Searching for the origins of musicality across species. Philosophical Transactions of the Royal Society B: Biological Sciences, 370(1664), 20140094.

Honing, H. (2012). Without it no music: beat induction as a fundamental musical trait. Annals of the New York Academy of Sciences, 1252(1), 85-91.

Honing, H., ten Cate, C., Peretz, I., Trehub, S.E. (2015). Without it no music: cognition, biology and evolution of musicality. Philosophical Transactions of the Royal Society B, 370: 20140088.

Howell, S., Schwandt, M., Fritz, J., Roeder, E., Nelson, C. (2003). A stereo music system as environmental enrichment for captive chimpanzees. Lab animal, 32(10), 31-36.

Hulse, S.H., Bernard, D.J., Braaten, R.F. (1995). Auditory discrimination of chordbased spectral structures by European starlings (Sturnus vulgaris). Journal of Experimental Psychology: General, 124(4), 409.

Hulse, S.H., Cynx, J. (1985). Relative pitch perception is constrained by absolute pitch in songbirds (Mimus, Molothrus, and Sturnus). Journal of Comparative Psychology, 99(2), 176.

Hulse, S.H., Takeuchi, A.H., Braaten, R.F. (1992). Perceptual invariances in the comparative psychology of music. Music Perception: An Interdisciplinary Journal, 10(2), 151-184.

Immordino-Yang, M.H., Singh, V. (2013). Hippocampal contributions to the processing of social emotions. Human brain mapping, 34(4), 945-955.

Izumi, A. (2000). Japanese monkeys perceive sensory consonance of chords. The Journal of the Acoustical Society of America, 108(6), 3073-3078.

Jones, M.R., Boltz, M. (1989). Dynamic attending and responses to time. Psychological review, 96(3), 459.

Jusczyk, P.W., Krumhansl, C.L. (1993). Pitch and rhythmic patterns affecting infants' sensitivity to musical phrase structure. Journal of experimental psychology: Human perception and performance, 19(3), 627.

Juslin, P.N., \& Laukka, P. (2003). Communication of emotions in vocal expression and music performance: Different channels, same code? Psychological bulletin, 129(5), 770.

Keehn, R.J.J., Iversen, J.R., Schulz, I., Patel, A.D. (2019). Spontaneity and diversity of movement to music are not uniquely human. Current Biology, 29(13), R621R622.

Kirschner, S., Tomasello, M. (2010). Joint music making promotes prosocial behavior in 4-year-old children. Evolution and Human Behavior, 31(5), 354-364.

Kniffin, K.M., Yan, J., Wansink, B., Schulze, W.D. (2017). The sound of cooperation: Musical influences on cooperative behavior. Journal of organizational behavior, 38(3), 372-390.

Koelsch, S., Fritz, T., v. Cramon, D.Y., Müller, K., Friederici, A.D. (2006). Investigating emotion with music: an fMRI study. Human brain mapping, 27(3), 239-250. 
Kogan, L.R., Schoenfeld-Tacher, R., Simon, A.A. (2012). Behavioral effects of auditory stimulation on kenneled dogs. Journal of Veterinary Behavior: Clinical Applications and Research, 7(5), 268-275.

Leboucher, G., Vallet, E., Nagle, L., Béguin, N., Bovet, D., Hallé F., Draganoiu, T., Amy, M., Kreutzer, M. (2012). Studying female reproductive activities in relation to male song: the domestic canary as a model. Advances in the Study of Behavior, 44, 183-223.

Le Covec, M., Aimé, C., Bovet, D. (2019). Combinatory sound object play in cockatiels: a forerunner of music?. Behaviour, 156(5-8), 1-23.

Lewkowicz, D.J. (2003). Learning and discrimination of audiovisual events in human infants: The hierarchical relation between intersensory temporal synchrony and rhythmic pattern cues. Developmental psychology, 39(5), 795.

Malloch, S.N. (1999). Mothers and infants and communicative musicality. Musicae scientiae, 3(1), 29-57.

Malloch, S., Trevarthen, C. (2009). Communicative musicality. Exploring the basis of buman.

Masataka, N. (1999). Preference for infant-directed singing in 2-day-old hearing infants of deaf parents. Developmental psychology, 35(4), 1001.

Masataka, N. (2006). Preference for consonance over dissonance by hearing newborns of deaf parents and of hearing parents. Developmental science, 9(1), 46-50.

McDermott, J.,H. Hauser, M.D. (2006). Thoughts on an empirical approach to the evolutionary origins of music. Music Perception: An Interdisciplinary Journal, 24(1), 111-116.

McDermott, J.H., Schultz, A.F., Undurraga, E.A., Godoy, R.A. (2016). Indifference to dissonance in native Amazonians reveals cultural variation in music perception. Nature, 535(7613), 547.

Miller, G. (2000). Evolution of human music through sexual selection. In N.L. Wallin, B. Merker, S. Brown (eds.), The origins of music (pp. 329-360). Cambridge, MA, US: The MIT Press.

Morton, J., Johnson, M. (1991). The perception of facial structure in infancy. In G.R. Lockhead \& J.R. Pomerantz (eds.), The perception of structure: Essays in honor of Wendell R. Garner (pp. 317-325).

Mosing, M.A., Madison, G., Pedersen, N.L., Kuja-Halkola, R., Ullén, F. (2014). Practice does not make perfect: no causal effect of music practice on music ability. Psychological science, 25(9), 1795-1803.

Mosing, M.A., Verweij, K.J., Madison, G., Pedersen, N.L., Zietsch, B.P., Ullén, F. (2015). Did sexual selection shape human music? Testing predictions from the sexual selection hypothesis of music evolution using a large genetically informative sample of over 10,000 twins. Evolution and Human Behavior, 36(5), 359-366.

Murray, I.R., Arnott, J.L. (1993). Toward the simulation of emotion in synthetic speech: A review of the literature on human vocal emotion. The Journal of the Acoustical Society of America, 93(2), 1097-1108.

Nakata, T., Trehub, S.E. (2004). Infants' responsiveness to maternal speech and singing. Infant Behavior and Development, 27(4), 455-464.

Nawrot, E.S. (2003). The perception of emotional expression in music: Evidence from infants, children and adults. Psychology of Music, 31(1), 75-92.

Nazzi, T., Floccia, C., Bertoncini, J. (1998). Discrimination of pitch contours by neonates. Infant Behavior and Development, 21(4), 779-784. 
North, A.C., Tarrant, M., Hargreaves, D.J. (2004). The effects of music on helping behavior: A field study. Environment and Behavior, 36(2), 266-275.

Odom, K.J., Hall, M.L., Riebel, K., Omland, K.E., Langmore, N.E. (2014). Female song is widespread and ancestral in songbirds. Nature Communications, 5, 3379.

Ota, N., Gahr, M., Soma, M. (2015). Tap dancing birds: the multimodal mutual courtship display of males and females in a socially monogamous songbird. Scientific reports, 5, 16614.

Otsuka, Y., Yanagi, J., Watanabe, S. (2009). Discriminative and reinforcing stimulus properties of music for rats. Behavioural Processes, 80(2), 121-127.

Palmer, C., Jungers, M.K., Jusczyk, P.W. (2001). Episodic memory for musical prosody. Journal of Memory and Language, 45(4), 526-545.

Papoušek, M. (1992). Early ontogeny of vocal communication in parent-infant interactions. In: Papoušek H, Jürgens U, Papoušek M (eds.) Nonverbal vocal communication: comparative and developmental approaches. New York: Cambridge University Press.

Patel, A.D., Iversen, J.R., Bregman, M.R., Schulz, I. (2009). Experimental evidence for synchronization to a musical beat in a nonhuman animal. Current biology, 19(10), 827-830.

Patel, A.D., Iversen, J.R., Rosenberg, J.C. (2006). Comparing the rhythm and melody of speech and music: The case of British English and French. The Journal of the Acoustical Society of America, 119(5), 3034-3047.

Plantinga, J., Trehub, S.E. (2014). Revisiting the innate preference for consonance. Journal of Experimental Psychology: Human Perception and Performance, 40(1), 40.

Péron, F., Hoummady, S., Mauny, N., Bovet, D. (2012). Touch screen device and music as enrichments to captive housing conditions of African grey parrots. Journal of Veterinary Behavior-Clinical Applications and Research, 7(6): e13.

Phillips-Silver, J., Trainor, L.J. (2005). Feeling the beat in music: Movement influences rhythm perception in infants. Science, 308, 1430.

Pinker, S. (1997). How the mind works. London: Allen Lane.

Poli, M., Previde, E.P. (1991). Discrimination of musical stimuli by rats (Rattus norvegicus). International Journal of Comparative Psychology, 5(1).

Porter, D., Neuringer, A. (1984). Music discriminations by pigeons. Journal of Experimental Psychology: Animal Behavior Processes, 10(2), 138.

Rabinowitch, T.C., Knafo-Noam, A. (2015). Synchronous rhythmic interaction enhances children's perceived similarity and closeness towards each other. PloS one, 10(4), e0120878.

Rabinowitch, T.C., Cross, I., Burnard, P. (2013). Long-term musical group interaction has a positive influence on empathy in children. Psychology of music, 41(4), 484-498.

Ramus, F., Nespor, M., Mehler, J. (1999). Correlates of linguistic rhythm in the speech signal. Cognition, 73(3), 265-292.

Ravignani, A., Bowling, D.L., Fitch, W. (2014). Chorusing, synchrony, and the evolutionary functions of rhythm. Frontiers in Psychology, 5, 1118.

Reinert, J. (1957). Akustische Dressurversuche an einem indischen Elefanten. Zeitschrift fuer Tierpsychologie, 14(1), 100-126.

Reinert, J. (1965). Takt und Rhythmusunterscheidung bei Dohlen Zeitschrift für Tierpsychologie, 22(6), pp. 623-671. doi: 10.1111/j.1439-0310.1965.tb01683.x 
Robbins, L., Margulis, S.W. (2014). The effects of auditory enrichment on gorillas. Zoo biology, 33(3), 197-203.

Roederer, J.G. (1984). The search for a survival value of music. Music Perception: An Interdisciplinary Journal, 1(3), 350-356.

Rothenberg, D., Roeske, T.C., Voss, H.U., Naguib, M., Tchernichovski, O. (2014). Investigation of musicality in birdsong. Hearing research, 308, 71-83.

Saliba, S., Esseily, R., Filippa, M., Kuhn, P., Gratier, M. (2018). Exposure to human voices has beneficial effects on preterm infants in the neonatal intensive care unit. Acta Paediatrica, 107(7), 1122-1130.

Schachner, A., Brady, T.F., Pepperberg, I.M., Hauser, M.D. (2009). Spontaneous motor entrainment to music in multiple vocal mimicking species. Current Biology, 19(10), 831-836.

Schellenberg, E.G., Trehub, S.E. (1994). Frequency ratios and the discrimination of pure tone sequences. Perception \& Psychophysics, 56(4), 472-478.

Scherer, K.R. (1986). Vocal affect expression: A review and a model for future research. Psychological bulletin, 99(2), 143.

Scherer, K.R. (1991). Emotion expression in speech and music. In Music, language, speech and brain (pp. 146-156). Palgrave, London.

Schmidt, L.A., Trainor, L.J. (2001). Frontal brain electrical activity (EEG) distinguishes valence and intensity of musical emotions. Cognition \& Emotion, 15(4), 487-500.

Searcy, W.A., Andersson, M. (1986). Sexual selection and the evolution of song. Annual Review of Ecology and Systematics, 17(1), 507-533.

Shenfield, T., Trehub, S.E., Nakata, T. (2003). Maternal singing modulates infant arousal. Psychology of Music, 31(4), 365-375.

Shinozuka, K., Ono, H., Watanabe, S. (2013). Reinforcing and discriminative stimulus properties of music in goldfish. Behavioural processes, 99, 26-33.

Soma, M., Mori, C. (2015). The songbird as a percussionist: syntactic rules for nonvocal sound and song production in Java sparrows. PLoS One, 10(5), e0124876.

Sonnenschein, E., Reyer, H.U. (1983). Mate-Guarding and other Functions of Antiphonal Duets in the Slate-coloured Boubou (Laniarius funebris) 1. Zeitschrift für Tierpsychologie, 63(2-3), 112-140.

Sutoo, D.E., Akiyama, K. (2004). Music improves dopaminergic neurotransmission: demonstration based on the effect of music on blood pressure regulation. Brain research, 1016(2), 255-262.

Tarr, B., Launay, J., Dunbar, R.I. (2014). Music and social bonding:"self-other" merging and neurohormonal mechanisms. Frontiers in Psychology, 5, 1096.

Thorpe, L.A., Trehub, S.E. (1989). Duration illusion and auditory grouping in infancy. Developmental Psychology, 25(1), 122.

Thorpe, L.A., Trehub, S.E., Morrongiello, B.A., Bull, D. (1988). Perceptual grouping by infants and preschool children. Developmental Psychology, 24(4), 484.

Trainor, L.J. (1996). Infant preferences for infant-directed versus noninfant-directed playsongs and lullabies. Infant behavior and development, 19(1), 83-92.

Trainor, L.J. (1997). Effect of frequency ratio on infants' and adults' discrimination of simultaneous intervals. Journal of Experimental Psychology: Human Perception and Performance, 23(5), 1427. 
Trainor, L.J., Heinmiller, B.M. (1998). The development of evaluative responses to music: Infants prefer to listen to consonance over dissonance. Infant Behavior and Development, 21(1), 77-88.

Trainor, L.J., Trehub, S.E. (1993). Musical context effects in infants and adults: Key distance. Journal of Experimental Psychology: Human Perception and Performance, $19(3), 615$.

Trainor, L.J., Tsang, C.D., Cheung, V.H. (2002). Preference for sensory consonance in 2-and 4-month-old infants. Music Perception: An Interdisciplinary Journal, 20(2), 187-194.

Trehub, S.E. (2003). Toward a developmental psychology of music. Annals of the New York Academy of Sciences, 999(1), 402-413.

Trehub, S.E. (2019). Multimodal music in infancy and early childhood. The Oxford Handbook of Music and the Body, 383.

Trehub, S.E., Becker, J., Morley, I. (2015). Cross-cultural perspectives on music and musicality. Philosophical Transactions of the Royal Society B: Biological Sciences, 370(1664), 20140096.

Trehub, S.E., Cohen, A.J., Thorpe, L.A., Morrongiello, B.A. (1986). Development of the perception of musical relations: Semitone and diatonic structure. Journal of Experimental Psychology: Human Perception and Performance, 12(3), 295.

Trehub, S.E., Hannon, E.E. (2006). Infant music perception: Domain-general or domain-specific mechanisms? Cognition, 100(1), 73-99.

Trehub, S.E., Nakata, T. (2001). Emotion and music in infancy. Musicae scientiae, 5(1_ suppl), 37-61.

Trehub, S.E., Thorpe, L.A., Trainor, L.J. (1990). Infants' perception of good and bad melodies. Psychomusicology: A Journal of Research in Music Cognition, 9(1), 5.

Trehub, S.E., Thorpe, L.A. (1989). Infants' perception of rhythm: Categorization of auditory sequences by temporal structure. Canadian Journal of Psychology/Revue canadienne de psychologie, 43(2), 217.

Trehub, S.E., Trainor, L. (1998). Singing to infants: Lullabies and play songs. Advances in infancy research, 12, 43-78.

Trehub, S.E., Unyk, A.M., Kamenetsky, S.B., Hill, D.S., Trainor, L.J., Henderson, J.L., Saraza, M. (1997). Mothers' and fathers' singing to infants. Developmental psychology, 33(3), 500.

Trehub, S.E., Unyk, A.M., Trainor, L.J. (1993). Maternal singing in cross-cultural perspective. Infant behavior and development, 16(3), 285-295.

Tunçgenç, B., Cohen, E. (2016). Movement synchrony forges social bonds across group divides. Frontiers in Psychology, 7, 782.

Tunçgenç, B., Cohen, E., Fawcett, C. (2015). Rock with me: The role of movement synchrony in infants' social and nonsocial choices. Child Development, 86(3), 976-984.

Ukkola-Vuoti, L., Kanduri, C., Oikkonen, J., Buck, G., Blancher, C., Raijas, P., ... Järvelä, I. (2013). Genome-wide copy number variation analysis in extended families and unrelated individuals characterized for musical aptitude and creativity in music. PLoS One, 8(2), e56356.

Unyk, A.M., Trehub, S.E., Trainor, L.J., Schellenberg, E.G. (1992). Lullabies and simplicity: A cross-cultural perspective. Psychology of Music, 20(1), 15-28. 
Videan, E.N., Fritz, J., Howell, S., Murphy, J. (2007). Effects of two types and two genres of music on social behavior in captive chimpanzees (Pan troglodytes). Journal of the American Association for Laboratory Animal Science, 46(1), 66-70.

Watanabe, S., Nemoto, M. (1998). Reinforcing property of music in Java sparrows (Padda oryzivora). Behavioural Processes, 43(2), 211-218.

Wells, D.L., Coleman, D., Challis, M.G. (2006). A note on the effect of auditory stimulation on the behaviour and welfare of zoo-housed gorillas. Applied Animal Behaviour Science, 100(3-4), 327-332.

Wells, D.L., Graham, L., Hepper, P.G. (2002). The influence of auditory stimulation on the behaviour of dogs housed in a rescue shelter. Animal Welfare, 11(4), 385-393.

Wells, D.L., Irwin, R.M. (2008). Auditory stimulation as enrichment for zoo-housed Asian elephants (Elephas maximus). Animal Welfare, 17(4), 335-340.

Zarco, W., Merchant, H., Prado, L., Mendez, J.C. (2009). Subsecond timing in primates: comparison of interval production between human subjects and rhesus monkeys. Journal of neurophysiology, 102(6), 3191-3202.

Zentner, M.R., Kagan, J. (1998). Infants' perception of consonance and dissonance in music. Infant Behavior and Development, 21(3), 483-492. 